\title{
MODEL THEORY FOR METRIC STRUCTURES
}

\author{
MWF at 2:00 \\ Math 595 (MTM) \\ Ward Henson \\ 347 Altgeld \\ Spring 2005
}

A metric structure is a many-sorted structure with each sort a metric space, which for convenience is assumed to have finite diameter. Additionally there are functions (of several variables) between sorts, assumed to be uniformly continuous. Examples include metric spaces themselves, measure algebras (with the metric $d(A, B)=\mu(A \Delta B)$, where $\Delta$ is symmetric difference), and structures based on Banach spaces (where one interprets the sorts as balls), including Banach lattices, $\mathrm{C}^{*}$ algebras, etc.

The usual first-order logic does not work very well for such structures, and several good alternatives have been developed. One is the logic of positive bounded formulas with an approximate semantics (see [3]). Another is the setting of compact abstract theories (cats) (see [1]). A recent development, which will be emphasized in this course, is the convergence of these two points of view and the realization that they are also connected to the $[0,1]$-valued continuous logic that was studied extensively in the 1960s and then dropped (see [2]). This leads to a new formalism for the logic of metric structures in which the operations of sup and inf play a central role.

The analogy between this logic for metric space structures and the usual first order logic for ordinary structures is far reaching. This new logic satisfies the compactness theorem, Löwenheim-Skolem theorems, diagram arguments, existence of saturated and homogeneous models, characterizations of quantifier elimination and model completeness, Beth's definability theorem, Craig's interpolation theorem, the omitting types theorem, fundamental results of stability theory, and appropriate analogues of essentially all results in basic model theory of first order logic.

The purpose of this course is to present this logic for metric structures as completely as possible, and to show its application to metric spaces, measure algebras, structures from functional analysis such as Banach lattices and $\mathrm{C}^{*}$-algebras, Hilbert space operators, and the like.

Prerequisites: The main prerequisite is readiness to do mathematics at the graduate level. No previous experience with logic or any other specific area of mathematics is expected, beyond what is included in the equivalent of a good undergraduate mathematics major. The motivation for many things done in the course will, however, be more clear to those who have studied some basic model theory and real analysis.

Text and References: There is no text; the instructor will provide notes and articles as background.

Student Projects: Students will be encouraged to do a project related to the content of the course. For parts of the course, students will be asked to work in groups to produce lecture notes.

References:

[1] I. Ben-Yaacov, Positive model theory and compact abstract theories, J. Math. Logic 3 (2003), $85-118$.

[2] C. C. Chang and H. J. Keisler, Continuous Model Theory, Princeton Univ. Press (1966).

[3] C. W. Henson and J. Iovino, Ultraproducts in Analysis, in Analysis and Logic, London Mathematical Society Lecture Notes 262, Cambridge University Press (2002), 1-113. 